\title{
The Effects of Continuous Positive Airway Pressure on Premature Ventricular Contractions and Ventricular Wall Stress in Patients with Heart Failure and Sleep Apnea
}

\author{
Sabri Seyis $\mathbb{D}^{\mathbb{D}},{ }^{1}$ Adnan Kazım Usalan, ${ }^{2}$ Ibrahim Rencuzogullari, ${ }^{3}$ Özge Kurmuş (D), ${ }^{4}$ \\ and Adil Can Gungen ${ }^{5}$ \\ ${ }^{1}$ Department of Cardiology, Live Istinye University Hospital, Istanbul, Turkey \\ ${ }^{2}$ Department of Chest Disease, Medical Park Hospital, Mersin, Turkey \\ ${ }^{3}$ Department of Cardiology, Kafkas University Faculty of Medicine, Kars, Turkey \\ ${ }^{4}$ Department of Cardiology, Ufuk University, Ankara, Turkey \\ ${ }^{5}$ Department of Chest Disease, Live Istinye University Hospital, Istanbul, Turkey \\ Correspondence should be addressed to Sabri Seyis; sabriseyis@yahoo.com
}

Received 7 October 2017; Revised 5 November 2017; Accepted 9 January 2018; Published 7 February 2018

Academic Editor: Alice M. Turner

Copyright $\odot 2018$ Sabri Seyis et al. This is an open access article distributed under the Creative Commons Attribution License, which permits unrestricted use, distribution, and reproduction in any medium, provided the original work is properly cited.

Background. We aimed to investigate the effects of continuous positive airway pressure (CPAP) treatment on electrocardiography (ECG), premature ventricular contraction load on 24-hour Holter recordings, and implantable cardioverter defibrillator (ICD) shocks in patients with obstructive sleep apnea syndrome (OSAS) and heart failure. Methods. Patients with heart failure and ICD and patients with newly diagnosed OSAS were divided into two groups according to CPAP treatment. To compare the impact of CPAP on ECG parameters, both baseline and 6-month ECG, 24-hour Holter ECG, ambulatory blood pressure monitoring, echocardiography, polysomnography, and laboratory parameters were collected. Results. CPAP treatment significantly reduced the frequency of premature ventricular contractions, T-peak to T-end, corrected QT, corrected QT dispersion, and T-peak to T-end/corrected QT ratio in the study group ( $p<0.001$ for all). Although the baseline NT-proBNP levels were similar between study and control groups, after six months, the NT-pro-BNP levels of the study group were significantly lower than that of the control group ( $39.18 \pm 7.57$ versus $46.11 \pm 7.65 ; p<0.001)$. Conclusions. CPAP treatment in patients with heart failure and ICD and in patients with newly diagnosed OSAS may have beneficial effects on premature ventricular contractions and electrocardiographic arrhythmia indices and NT-pro-BNP levels. However, these results are needed to be clarified with further studies.

\section{Introduction}

Obstructive sleep apnea syndrome (OSAS) is characterized by temporary airway collapse during sleep and affects more than $4 \%$ of men and $2 \%$ of women [1]. Many cardiovascular diseases have been found to be associated with OSAS including arterial hypertension, ischemic heart disease, heart failure, arrhythmias, and stroke. Cardiovascular morbidity and mortality have been shown to be high in patients with OSAS $[2,3]$. Sympathetic overactivity, activation of inflammatory cascades, vascular endothelial dysfunction, abnormalities in the coagulation pathway, and metabolic dysregulation are likely to be involved in the pathogenesis of the cardiovascular complications of OSAS.

The relation between the congestive heart failure and OSAS is somewhat complex. OSAS may affect cardiac functions and contribute to the development of heart failure in the long-term. On the other hand, heart failure itself also plays a role in the pathogenesis of OSAS [4-7]. Several studies reported that continuous positive airway pressure (CPAP) treatment in patients with congestive heart failure and OSAS reduced sympathetic activation and improved left 
ventricular ejection fraction [8-10]. However, the positive effect of CPAP treatment on the cardiovascular system is unclear in terms of the prognosis of patients with congestive heart failure and OSAS [11].

There are some arrhythmic changes on electrocardiography (ECG) in patients with OSAS, and some improvement with CPAP therapy has been reported [12-14]. The association between arrhythmias and OSAS is well known $[15,16]$. OSAS associated with complex ventricular ectopia and ventricular tachycardia has been documented in a previous study [17]. CPAP treatment also resulted in a 58\% reduction in premature ventricular beats [18]. Those findings may help to explain the pathogenesis of sudden death in OSAS patients [19]. It has also been shown that OSAS patients have a 4-fold higher frequency of atrial fibrillation if apnoeahypopnoea index (AHI) index is greater than 30 [17].

Heart failure is a life-threatening disease with a growing incidence in developed countries due to better treatment options for heart failure, improvement of survival after myocardial infarction, and increased life expectancy. The introduction of implantable devices such as implantable cardioverter defibrillators (ICD) has improved the overall survival of patients with heart failure [20]. ICD treatment reduces mortality in patients with heart failure. However, certain conditions such as drug-resistant ventricular arrhythmias and atrial fibrillation may increase morbidity and mortality due to inappropriate and frequent shocks.

The aim of the present study is to investigate whether the regular CPAP treatment improves ECG findings and reduces premature ventricular contractions (PVC) and ICD shocks in patients with OSAS, cardiac failure, PVC, and ICD implant.

\section{Materials and Methods}

2.1. Study Population. This study is an observational study conducted with patients followed at the Department of Cardiology with diagnosis of chronic heart failure. Our inclusion criteria for the study population were ejection fraction $<35 \%$, stable NYHA class $\geq$ II, the presence of ICD, sinus rhythm, and $>30 \mathrm{PVC} /$ hour on 24-hour Holter monitorization. In addition, patients had to be on stable heart failure medication for at least 4 weeks prior to enrolment and during follow-up. Patients filled Epworth Sleepiness Scale (ESS) form. Clinically suspected patients were referred to Department of Chest Diseases for possible OSAS diagnosis. After polysomnographic study in the sleep laboratory, CPAP treatment was recommended for moderate to severe OSAS patients. Patients only with OSAS were included, and patients with central sleep apnea (CSA) and mixed apnea were not included. Protocol of the study was approved by the local ethics committee, and subjects involved in the study signed informed consent approved by the institution.

Baseline ECG, 24-hour Holter ECG, ambulatory blood pressure monitoring, echocardiography, laboratory investigations, and ICD measurements were recorded. Age, sex, body mass index (BMI), the cause of heart failure, heart rate, arterial blood pressure, left ventricular end diastolic diameter (LVEDD), left atrium (LA) diameter, polysomnography findings, levels of creatinine, and hemoglobin of the participants were also recorded. Class of drugs used by the patient was also recorded. Study group $(n=40)$ consisted of ICD implanted heart failure and OSAS patients who accepted CPAP treatment, and control group $(n=40)$ consisted of ICD implanted heart failure and OSAS patients who did not accept CPAP treatment. Chronic atrial fibrillation, bundle branch block, application of cardiac resynchronization therapy (CRT), the presence of pacemaker rhythm, estimated glomerular filtration rate of less than $60 \mathrm{~mL} / \mathrm{min} / 1.73 \mathrm{~m}^{2}$ (CKD stage $\geq$ III), significant lung disease, thyroid dysfunction, and New York Heart Association (NYHA) grade IV heart failure were the exclusion criteria. Six months after the initiation of CPAP therapy, ECG, 24-hour Holter monitorization, ambulatory blood pressure monitoring, and echocardiography were repeated in both groups.

2.2. Polysomnography. All referred individuals with heart failure and suspected OSAS were subjected to 1 full-night polysomnography at the sleep laboratory using the Nox A1 PSG System (Nox Medical, Iceland). Physiological variables evaluated during polysomnography included electroencephalogram, electrooculogram, electromyogram (submentonian and tibialis), electrocardiogram, oral/nasal airflow measured by oronasal thermistors, respiratory effort (thorax and abdomen), body position, and blood oxygen saturation $\left(\mathrm{SpO}_{2}\right)$ (mean and minimum $\mathrm{SpO}_{2}$ ). The exam was performed according to specific criteria for the definition of sleep stages [21] by trained technicians who were unaware of the patient characteristics.

Sleep-related respiratory events and arousals were scored according to the American Academy of Sleep Medicine Manual for Scoring Sleep and Associated Events [22]. Central sleep apnea was defined as the absence of thoracic and abdominal movements with absence of airflow. Obstructive sleep apnea was defined as the absence of airflow in the presence of thoracic and abdominal movements. Mixed apnea was defined as apnea without any thoracoabdominal movements in whom thoracoabdominal movements reoccurred prior to reoccurrence of nasal flow. Apnea was defined as complete cessation of the airflow $\geq 10 \mathrm{~s}$. Hypopnea was defined as $\geq 30 \%$ decrease in airflow from baseline for at least 10 seconds with $a \geq 3 \%$ oxygen desaturation from preevent baseline, and/or the event is associated with an arousal. Apnea/hypopnea index was determined via careful calculations. AHI score between 15 and 29 was considered as OSAS with moderate severity. Severe OSAS was defined as $\mathrm{AHI} \geq 30$.

2.3. Treatment. Autotitrating CPAP was used in the sleep laboratory within a period of less than 7 days after the diagnostic study to get a fixed CPAP pressure value. The fixed CPAP pressure was used for the rest of the study. All patients were scheduled for follow-up 2 weeks after randomization and, subsequently, at $4,8,12,18$, and 24 weeks. During the follow-up visits, adherence to CPAP and antihypertensive 
medications was documented. Compliance with CPAP therapy was based on the self-report of patients at the end of the study period. The use ratio of CPAP (\%) during the study period, which was the average time with CPAP to total time spent in bed, was $91 \%$ (range $72-100 \%$ ).

2.4. Electrocardiography. The 12-lead ECG (CARDIOVIT AT-102 Plus, Schiller, Switzerland) was recorded at a paper speed of $25 \mathrm{~mm} / \mathrm{s}$ at $10 \mathrm{~mm} / \mathrm{mV}$ amplitude in the supine position. ECGs were performed while the patient was at rest and at the morning hours (between 8:00 and 10:00 AM). All of the ECGs were scanned with a resolution of $800 \mathrm{dpi}$ and transferred to a personal computer. ECG measurements of QT and Tp-e intervals at V5 were performed by experienced cardiologists who were blinded to the patient data. If V5 was not suitable, V4 or V6 were measured, respectively. A mean value of three readings was calculated for each lead. The QT interval was measured from the beginning of the QRS complex to the end of the Twave. To adjust QT for heart rate, we calculated QTc according to Bazett's formula QTc $=\mathrm{QT} / \sqrt{\mathrm{R}} \overline{\mathrm{R}}$, where RR is the RR interval in seconds. The Tp-e interval was defined as the interval from the peak of $\mathrm{T}$ wave to the end of $\mathrm{T}$ wave. The Tp-e/corrected QT ratio was calculated from these measurements. QTc dispersion is determined as the difference between the maximum and minimum QTc in all considered leads. All ECG measurements were performed by a cardiologist who was blinded to other patient information.

2.5. Echocardiographic Evaluation. We used a 2-dimensional, M-mode cardiovascular ultrasound system (Vingmed Vivid 7 system GE, Germany) for echocardiographic evaluation. The examinations were performed in the left lateral decubitus position. Parasternal long- and short-axis views and apical views were used as standard imaging windows. We measured left atrial and left ventricular end systolic diameters from parasternal long-axis view. Ejection fraction was calculated by using modified Simpson method. All echocardiographic examinations were performed by an experienced cardiologist who was blinded to patient data.

2.6. Holter Assessment. Holter ECG (medilog ${ }^{\circledR}$ AR4 plus Holter system, Schiller, Switzerland) recordings were analyzed using a MARS 8000 Holter scanner (GE Medical Systems, Milwaukee, Wisconsin). We determined all PVC, atrial fibrillation (AF), and ventricular tachycardia (VT) episodes. The percentage of PVCs was calculated as the total number of ventricular ectopic beats/the total number of beats recorded during 24 hour Holter ECG monitoring.

2.7. Ambulatory Blood Pressure Monitoring. Ambulatory blood pressure monitoring was carried for $24 \mathrm{~h}$ using an automated sphygmomanometer (BR-102 PLUS PWA, Schiller, Switzerland). The blood pressure was recorded every $20 \mathrm{~min}$ during the $24 \mathrm{~h}$ period. Mean values were recorded before and after CPAP treatment.
2.8. Blood Sampling and Analysis. Fasting blood samples were collected from an antecubital vein in the morning and were centrifuged within one hour. Serum samples were stored at $-80^{\circ} \mathrm{C}$ pending later measurements. N-terminal pro-brain natriuretic peptide (NT-proBNP) was measured by electrochemiluminescent immunoassay (Roche Diagnostics, Basel, Switzerland). Creatinine and hemoglobin levels were measured by standard laboratory methods. Serum NT-proBNP level was measured at baseline and at the end of 6-month follow-up in both groups. The estimated glomerular filtration rate $\left(\mathrm{mL} / \mathrm{min}\right.$ per $\left.1.73 \mathrm{~m}^{2}\right)$ was calculated to exclude renal dysfunction as a cause for increased NT-proBNP concentrations [23].

2.9. Statistical Analysis. All data were entered into a spreadsheet, and statistical analyses were performed by using $\mathrm{R}$ 3.3.2v (open source). Data were shown as mean \pm standard deviation for continuous variables, median (minimum-maximum) for ordinal ones, and frequency with percent for categorical ones. Comparisons for continuous variables were made by using independent samples $t$-test and for categorical variables Fisher's exact test. Normally distributed variables were compared with paired $t$-test and nonnormally distributed variables were compared with Mann-Whitney $U$ test for intragroup and intergroup differences. Two-Way Repeated Measures of ANOVA was also used for intragroup comparisons. A $p$ value less than 0.05 was considered statistically significant.

\section{Results}

The mean age of the study group was $58.4 \pm 8.14$ years and the control group was $59.18 \pm 7.97$ years. The female/male ratio in the control group was $8 / 32$ and in the control group was $9 / 31$. There was no significant difference between the groups in terms of age and sex $(p=0.67$ and $p=0.79)$. Baseline characteristics and other demographics of the study and control groups are summarized in Table 1.

Other baseline characteristics including BMI, ejection fraction, AHI, oxygen saturation, heart rate, and blood pressure were also similar between both groups $(p>0.05$ for all). Drugs used by the patients in both groups are summarized in Table 2. Drug usage characteristics of the study and control groups were also similar. No medicational change was done during follow-up especially in terms of antiarrhythmic drugs.

ESS values were similar between both groups (10.51 \pm 4.82 versus $10.73 \pm 5.23, p=0.74$ ).

At the end of 6 months of CPAP treatment, frequency of PVC, Tp-e, QTc, QTc dispersion, and Tp-e/QTc ratio decreased significantly in the study group $(p<0.001$ for all). These parameters did not change significantly in the control group after 6 months $(p>0.05$ for all). Table 3 summarizes the chances of frequency of PVC, Tp-e, QTc, QTc dispersion, and Tp-e/QTc ratio during the follow-up period in both groups.

CPAP treatment significantly reduced the heart rate and systolic and diastolic blood pressure and increased the 
TABLE 1: Baseline characteristics and other demographics of the study and control groups.

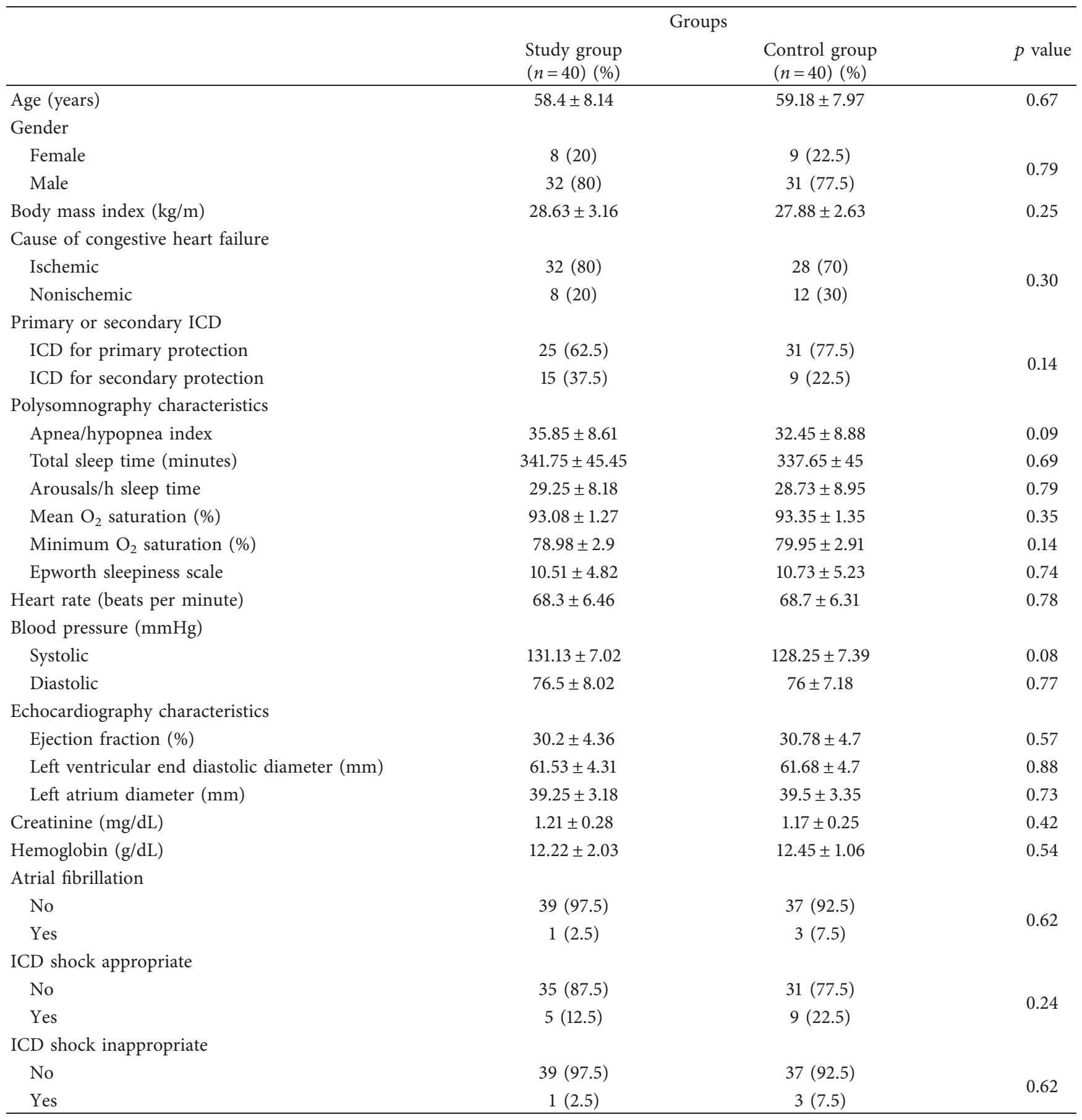

ejection fraction in the study group ( $p<0.05$ for all). BMI and LVEDD did not change significantly after 6 months of CPAP treatment. Table 4 summarizes the BMI, ejection fraction, heart rate, blood pressure, and LVEDD measures of the groups in detail.

Appropriate ICD shocks during follow-up in study and control groups due to VT/VF were seen in 5 patients and 9 patients, respectively ( $\% 12.5$ versus $\% 22.5, p=0.24)$. Inappropriate ICD shocks were much more in control group (\%2.5 versus $\% 7.5, p=0.62$ ) but did not reach statistical significance. Also atrial fibrillation rate detected by ICD devices was higher in control group but again did not have statistical significance.

Baseline NT-Pro BNP levels were $43.88 \pm 7.79 \mathrm{pg} / \mathrm{mL}$ in the study group and $44.65 \pm 7.79 \mathrm{pg} / \mathrm{mL}$ in the control group. The NT-Pro BNP levels at 6th month were $39.18 \pm$ $7.57 \mathrm{pg} / \mathrm{mL}$ in the study group and $46.11 \pm 7.65 \mathrm{pg} / \mathrm{mL}$ in the control group. There was a statistically significant difference between baseline and 6th-month measurements in the study group in terms of NT-Pro BNP $(p<0.001)$. There was also a statistically significant difference between the study and control groups at 6th month $(p<0.001)$, but not 
TABLE 2: Drug usage characteristics of the groups.

\begin{tabular}{|c|c|c|c|}
\hline & \multicolumn{2}{|c|}{ Groups } & \multirow[t]{2}{*}{$p$ value } \\
\hline & Study group $(n=40)$ & Control group $(n=40)$ & \\
\hline \multicolumn{4}{|l|}{$\beta$ blocker } \\
\hline Not using & $3(7.5)$ & $3(7.5)$ & \multirow{2}{*}{1.0} \\
\hline Using & $37(92.5)$ & $37(92.5)$ & \\
\hline \multicolumn{4}{|c|}{ Renin-angiotensin system blocker } \\
\hline Not using & $2(5)$ & $2(5)$ & \multirow{2}{*}{1.0} \\
\hline Using & $38(95)$ & $38(95)$ & \\
\hline \multicolumn{4}{|c|}{ Aldosterone antagonist } \\
\hline Not using & $9(22.5)$ & $9(22.5)$ & \multirow{2}{*}{1.0} \\
\hline Using & $31(77.5)$ & $31(77.5)$ & \\
\hline \multicolumn{4}{|c|}{ Class III antiarrhythmic agents } \\
\hline Not using & $25(62.5)$ & $30(75)$ & \multirow{2}{*}{0.23} \\
\hline Using & $15(37.5)$ & $10(25)$ & \\
\hline \multicolumn{4}{|l|}{ Diuretics } \\
\hline Not using & $5(12.5)$ & $3(7.5)$ & \multirow{2}{*}{0.71} \\
\hline Using & $35(87.5)$ & $37(92.5)$ & \\
\hline
\end{tabular}

TABLE 3: The chances of frequency of PVC, Tp-e, QTc, QTc dispersion, and Tp-e/QTc ratio during the follow-up period in both groups.

\begin{tabular}{|c|c|c|c|c|c|c|c|c|}
\hline & \multicolumn{3}{|c|}{ Study group } & \multicolumn{3}{|c|}{ Control group } & \multicolumn{2}{|c|}{$\begin{array}{l}\text { Study group versus } \\
\text { control group }\end{array}$} \\
\hline & Baseline & 6th month & $p$ value* & Baseline & 6th month & $p$ value ${ }^{*}$ & $\begin{array}{l}\text { Baseline } \\
p \text { value }\end{array}$ & $\begin{array}{c}\text { 6th month } \\
p \text { value }\end{array}$ \\
\hline Frequency of PVC & $1290(865-1665)$ & $945(685-1330)$ & $<0.001$ & $1360(960-1765)$ & $1325(990-1740)$ & 0.57 & 0.82 & $0.004^{* *}$ \\
\hline Tp-e interval (ms) & $79.7 \pm 7.02$ & $71.85 \pm 6.07$ & $<0.001$ & $79.18 \pm 8.01$ & $79.13 \pm 7.97$ & 0.89 & 0.76 & $<0.001$ \\
\hline QTc interval (ms) & $410.05 \pm 11.2$ & $397.2 \pm 11.64$ & $<0.001$ & $410.43 \pm 12.11$ & $414.25 \pm 11.56$ & 0.004 & 0.89 & $<0.001$ \\
\hline QTc dispersion & $53.28 \pm 8.51$ & $37.95 \pm 8.31$ & $<0.001$ & $55.48 \pm 9.97$ & $54.83 \pm 9.58$ & 0.16 & 0.29 & $<0.001$ \\
\hline Tp-e/QTc ratio & $194.18 \pm 14.99$ & $180.48 \pm 13.89$ & $<0.001$ & $192.15 \pm 15.03$ & $190.48 \pm 16.81$ & 0.13 & 0.55 & $<0.001$ \\
\hline
\end{tabular}

${ }^{*}$ Two-way repeated measures ANOVA was used; ${ }^{* *}$ frequency of premature ventricular contractions were compared between the groups using MannWhitney $U$ test; PVC: premature ventricular contractions; Tp-e: T-peak to T-end interval; QTc: corrected QT interval.

TABLE 4: The comparisons of certain parameters of the groups during the follow-up period.

\begin{tabular}{|c|c|c|c|c|c|c|}
\hline & \multicolumn{2}{|c|}{ Study group } & \multicolumn{4}{|c|}{ Control group } \\
\hline & Baseline & 6th month & $p$ value & Baseline & 6th month & $p$ value \\
\hline Body mass index $\left(\mathrm{kg} / \mathrm{m}^{2}\right)$ & $28.63 \pm 3.16$ & $28.65 \pm 2.92$ & 0.812 & $27.88 \pm 2.63$ & $27.83 \pm 2.54$ & 0.534 \\
\hline Ejection fraction (\%) & $30.2 \pm 4.36$ & $30.98 \pm 4.19$ & $<0.001$ & $30.78 \pm 4.7$ & $30.75 \pm 4.49$ & 0.860 \\
\hline Heart rate (beats per minute) & $68.3 \pm 6.46$ & $67.38 \pm 6.59$ & 0.002 & $68.7 \pm 6.31$ & $68.48 \pm 6.26$ & 0.060 \\
\hline Systolic blood pressure (mmHg) & $131.13 \pm 7.02$ & $127.5 \pm 5.66$ & $<0.001$ & $128.25 \pm 7.39$ & $127.75 \pm 7.16$ & 0.160 \\
\hline Diastolic blood pressure & $76.5 \pm 8.02$ & $74.13 \pm 6.59$ & $<0.001$ & $76 \pm 7.18$ & $76 \pm 7.18$ & 0.999 \\
\hline Left ventricular end diastolic diameter (mm) & $61.53 \pm 4.31$ & $61.68 \pm 4.32$ & 0.262 & $61.68 \pm 4.7$ & $62.03 \pm 4.65$ & 0.001 \\
\hline
\end{tabular}

baseline $(p=0.112)$ NT-Pro BNP measurements. Figure 1 shows the baseline and 6th-month NT-pro-BNP levels in the groups.

\section{Discussion}

Individuals with OSAS have frequent cessation or reduction of airflow during sleep that results in hypoxemia and arousals from sleep. Continuous positive airway pressure (CPAP) is the standard first-line treatment for OSAS [24].
One metaanalysis from consistent evidence from good- and fair-quality randomized controlled trials reported that CPAP effectively reduces AHI and excessive sleepiness in patients with OSAS [25]. Several studies showed the beneficial effects of CPAP on cardiac functions in OSAS patients $[26,27]$. The possible mechanism of action of CPAP may include improved myocardial oxygen delivery, decreased sympathetic activity, left ventricular transmural pressure, and afterload [28]. In the present study, we have found beneficial effects of CPAP treatment in patients with OSAS 


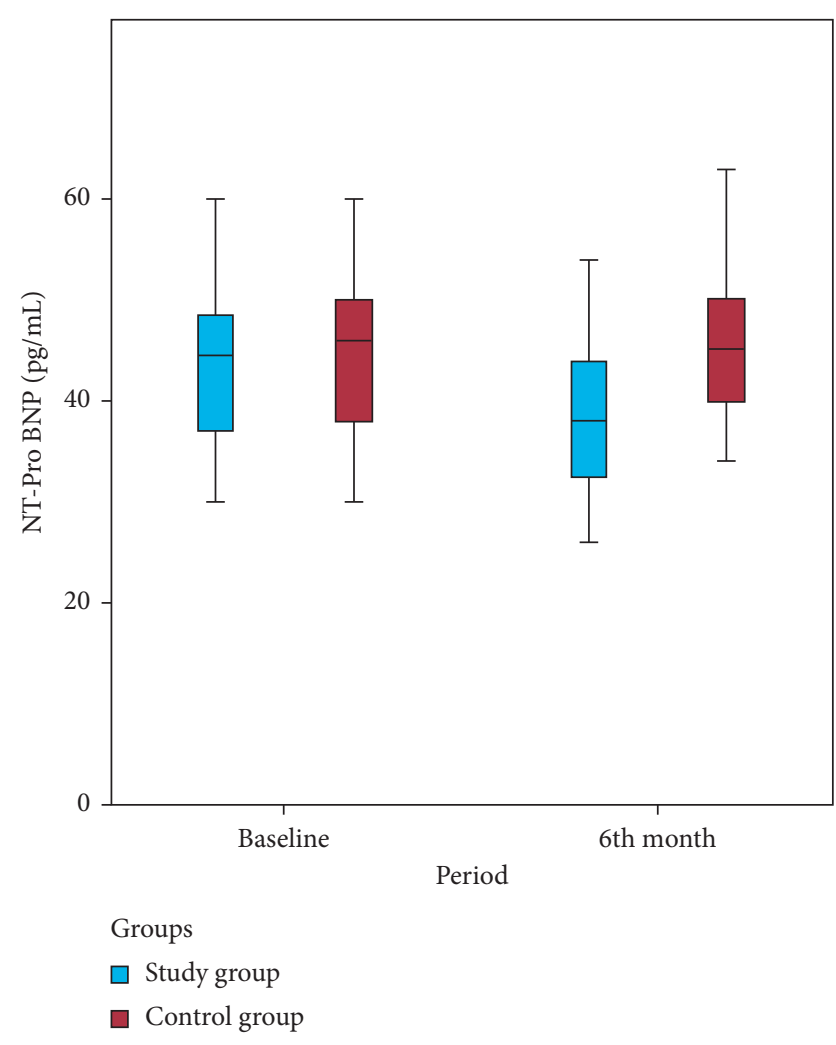

Figure 1: The baseline and 6-month NT-pro BNP levels in both groups.

and heart failure in terms of reduction in premature ventricular contractions and ventricular wall stress.

The issue of whether OSAS is an independent risk factor for patients with heart failure is controversial. The results of published studies on this subject vary significantly $[29,30]$. Lanfranchi et al. [29] reported approximately 4 -fold increased mortality rates in patients with OSAS and heart failure compared to heart failure patients without OSAS. Fries et al. [30] investigated the prognostic value of OSAS in congestive heart failure patients with ICD implants and found that mortality rates were $44 \%$ in central sleep apnea and $13 \%$ in the absence of apnea at the 2 years of follow-up $(p<0.05)$, but this study found no association between sleep apnea and appropriate ICD therapy for VT or VF. Adlakha and Shepard [31] speculated that hypoxia in the obstructive sleep apnea leads to cardiac arrhythmias. Sudden deaths, particularly at night, in OSAS may be due to these arrhythmias [19, 32]. Clay et al. [33] reported that OSAS patients with ICD had higher rates of ventricular ectopia and nonsustained ventricular tachycardia. Javaheri [34] found an increased frequency of VT and VES in patients with congestive heart failure and OSAS. Bitter et al. [35] speculated that OSAS is an independent risk factor for malignant ventricular arrhythmias that require ICD treatment. We found reduced premature ventricular contraction rates after 6 months of CPAP treatment in patients with OSAS and heart failure compared to untreated controls. We did not make major changes in the heart failure treatment of patients. So, favorable results would be attributable to CPAP treatment.
Ventricular repolarization and arrhythmogenesis can be assessed by several electrocardiographic parameters including QTc interval, QT dispersion, and $\mathrm{T}$ wave measurements [36]. Tp-e is used as an indicator of transmural dispersion of ventricular repolarization [37]. Prolonged Tp-e interval may predict ventricular arrhythmias and mortality $[38,39]$. Thus, Tp-e/QTc ratio has been proposed to be a more specific index of ventricular arrhythmogenesis [40, 41]. Kilicaslan et al. [36] reported prolonged Tp-e interval and increased Tp-e/QTc ratio in patients with moderate and severe OSAS as compared to healthy controls. Their finding of Tp-e and QTc interval in OSAS patients were comparable with the present study results. We found that, CPAP treatment significantly reduced the frequency of PVC, Tp-e interval, QTc interval, QTc dispersion, and Tp-e/QTc ratio in the study group, whereas the control group did not show any significant difference for the mentioned parameters.

Myocytes of cardiac ventricle constitute the major source of BNP-related neurohormones. B-type natriuretic peptide (BNP) is a 32 amino acid peptide primarily synthesized, stored, and secreted from left ventricle. NT proBNP is derived from the BNP and secreted when the ventricular wall stress increased [42, 43]. It has been used as an indicator of heart failure and symptomatic idiopathic PVC [42, 44, 45]. NT proBNP suggested as a sensitive indicator of PVCinduced increased ventricular wall stress [44, 45]. Skranes et al. [46] reported that the higher levels of NT-proBNP levels are independently associated with the incidence of frequent ventricular ectopy and complex ventricular ectopy in a moderately large, community-based population. Another study [47] found that fatigue was associated with higher baseline NT-proBNP in patients with frequent PVCs and preserved LV function. Tasci et al. [48] reported that nasal CPAP treatment significantly reduced NT-proBNP in patients with normotensive and hypertensive OSAS. Similarly, Strehmel et al. reported reduced NT-proBNP levels after CPAP treatment in patients with OSAS and coronary artery disease [49]. In the present study, we have found that 6 months CPAP treatment for OSAS significantly reduced the NT-proBNP levels which means reduced ventricular wall stress.

The present study has some limitations. We did not evaluate the AHI index using polysomnography at the 6-month followup. Therefore, an association between AHI and cardiac functions remained unclarified. The randomization in this study depended on the patient preferences (whether or not CPAP treatment is desired). However, real randomization in this context carries ethical considerations. The relatively small number of patients in our study is another limitation. A larger patient population with a longer follow-up would provide more precise results.

\section{Conclusions}

We have found that the frequency of PVC and NT-proBNP levels can be reduced by CPAP treatment in patients with heart failure and OSAS. CPAP therapy has been shown to significantly improve cardiac functions. Further studies are needed to clarify the possible relationships between CPAP and cardiac functions in detail. 


\section{Conflicts of Interest}

The authors declare that they have no conflicts of interest.

\section{References}

[1] T. Young, M. Palta, J. Dempsey et al., "The occurrence of sleepdisordered breathing among middle-aged adults," New England Journal of Medicine, vol. 328, no. 17, pp. 1230-1235, 1993.

[2] M. Butt, G. Dwivedi, O. Khair, and G. Y. H. Lip, "Obstructive sleep apnea and cardiovascular disease," International Journal of Cardiology, vol. 139, no. 1, pp. 7-16, 2010.

[3] W. T. McNicholas and M. R. Bonsigore, "Management Committee of EU COST ACTION B26. Sleep apnoea as an independent risk factor for cardiovascular disease: current evidence, basic mechanisms and research priorities," European Respiratory Journal, vol. 29, no. 3, pp. 156-178, 2007.

[4] M. R. Pinski, "Sleeping with the enemy: the heart in obstructive sleep apnea," Chest, vol. 121, no. 4, pp. 1022-1024, 2002.

[5] T. D. Bradley, "Floras, sleep apnea and heart failure. Part I: obstructive sleep apnea," Circulation, vol. 107, no. 12, pp. 16711678, 2003.

[6] M. T. Naughton, "Heart failure and obstructive apnea," Sleep Medicine Reviews, vol. 2, no. 2, pp. 93-103, 1998.

[7] S. M. Caples, R. Wolk, and V. K. Somers, "Influence of cardiac function and failure on sleep- disordered breathing: evidence for a causative role," Journal of Applied Physiology, vol. 99, no. 6, pp. 2433-2439, 2005.

[8] Y. Kaneko, JS. Floras, K. Usui et al., "Cardiovascular effects of continuous positive airway pressure in patients with heart failure and obstructive sleep apneas," New England Journal of Medicine, vol. 348, no. 13, pp. 1233-1241, 2003.

[9] D. R. Mansfield, N. C. Gollogly, D. M. Kaye et al., "Controlled trial of continuous positive airway pressure in obstructive sleep apnea and heart failure," American Journal of Respiratory and Critical Care Medicine, vol. 169, no. 3, pp. 361366, 2004.

[10] S. Malone, P. P. Liu, R. Holloway, R. Rutherford, A. Xie, and T. Douglas Bradley, "Obstructive sleep apnoea in patients with dilated cardiomyopathy: effects of continuous positive airway pressure," The Lancet, vol. 338, no. 8781, pp. 14801484, 1991.

[11] T. Roebuck, P. Solin, D. M. Kaye, P. Bergin, M. Bailey, and M. T. Naughton, "Increased long-term mortality in heart failure due to sleep apnoea is not yet proven," European Respiratory Journal, vol. 23, no. 5, pp. 735-740, 2004.

[12] I. Can, K. Aytemir, A. U. Demir et al., "P-wave duration and dispersion in patients with obstructive sleep apnea," International Journal of Cardiology, vol. 133, no. 3, pp. e85-e89, 2009.

[13] M. T. Maeder, T. Münzer, H. Rickli et al., "Association between heart rate recovery and severity of obstructive sleep apnea syndrome," Sleep Medicine, vol. 9, no. 7, pp. 753-761, 2008.

[14] K. Sumi, K. Chin, K. Takahashi et al., "Effect of nCPAP therapy on heart rate in patients with obstructive sleep apnoea-hypopnoea," QJM, vol. 99, no. 8, pp. 545-553, 2006.

[15] B. Phillips, "Sleep-disordered breathing and cardiovascular disease," Sleep Medicine Reviews, vol. 9, no. 2, pp. 131-140, 2006.

[16] A. S. Shamsuzzaman, B. J. Gersh, and V. K. Somers, "Obstructive sleep apnea. Implications for cardiac and vascular disease," JAMA, vol. 290, no. 14, pp. 1906-1914, 2003.

[17] R. Mehra, E. J. Benjamin, E. Shahar et al., "Association of nocturnal arrhythmias with sleep- disordered breathing: the sleep heart health study," American Journal of Respiratory and Critical Care Medicine, vol. 173, no. 8, pp. 910-916, 2006.

[18] C. M. Ryan, K. Usui, J. S. Floras et al., "Effect of continuous positive airway pressure on ventricular ectopy in heart failure patients with obstructive sleep apnoea," Thorax, vol. 60, no. 9, pp. 781-785, 2005.

[19] A. S. Gami, D. E. Howard, E. J. Olson, and V. K. Somers, "Day-night pattern of sudden death in obstructive sleep apnea," New England Journal of Medicine, vol. 352, no. 12, pp. 1206-1214, 2005.

[20] D. O. Verschure, B. L. van Eck-Smit, G. A. Somsen, R. J. Knol, and H. J. Verberne, "Cardiac sympathetic activity in chronic heart failure: cardiac (123)I-mIBG scintigraphy to improve patient selection for ICD implantation," Netherlands Heart Journal, vol. 24, no. 12, pp. 701-708, 2016.

[21] A. Rechtschaffen and A. Kales, A Manual of Standardized Terminology, Techniques and Scoring System for Sleep Stages of Human Subjects, University of California, Los Angeles, CA, USA, 1968.

[22] C. Iber, S. Ancoli-Israel, A. L. Chesson, and S. F. Quan, The AASM Manual for the Scoring of Sleep and Associated Events: Rules, Terminology and Technical Specifications, American Academy of Sleep Medicine, Westchester, IL, USA, 1st edition, 2007.

[23] S. Vickery, C. P. Price, R. I. John et al., "B-type natriuretic peptide (BNP) and amino-terminal proBNP in patients with CKD: relationship to renal function and left ventricular hypertrophy," American Journal of Kidney Diseases, vol. 46, no. 4, pp. 610-620, 2005.

[24] A. Y. Avidan, "The development of central sleep apnea with an oral appliance," Sleep Medicine, vol. 7, no. 1, pp. 85-86, 2006.

[25] D. E. Jonas, H. R. Amick, C. Feltner et al., Screening for Obstructive Sleep Apnea in Adults: An Evidence Review for the U.S. Preventive Services Task ForceEvidence Synthesis No. 146. AHRQ Publication No. 14-05216-EF-1, Agency for Healthcare Research and Quality, Rockville, MD, USA, 2017.

[26] T. D. Bradley and J. S. Floras, "Pathophysiologic and therapeutic implications of sleep apnea in congestive heart failure," Journal of Cardiac Failure, vol. 2, no. 3, pp. 223-240, 1996.

[27] J. W. Fung, T. S. T. Li, D. K. L. Choy et al., "Severe obstructive sleep apnea is associated with left ventricular diastolic dysfunction," Chest, vol. 121, no. 2, pp. 422-429, 2002.

[28] N. Dursunoglu, D. Dursunoglu, S. Ozkurt et al., "Effects of CPAP on right ventricular myocardial performance index in obstructive sleep apnea patients without hypertension," Respiratory Research, vol. 7, no. 1, p. 22, 2006.

[29] P. A. Lanfranchi, A. Braghiroli, E. Bosimini et al., "Prognostic value of nocturnal Cheyne- Stokes respiration in chronic heart failure," Circulation, vol. 99, no. 11, pp. 1435-1440, 1999.

[30] R. Fries, D. Bauer, A. Heisel et al., "Clinical significance of sleep-related breathing disorders in patients with implantable cardioverter defibrillators," Pacing and Clinical Electrophysiology, vol. 22, no. 1, pp. 223-227, 1999.

[31] A. Adlakha and J. W. Shepard, "Cardiac arrhythmias during normal sleep and in obstructive sleep apnea syndrome," Sleep Medicine Reviews, vol. 2, no. 1, pp. 45-60, 1998.

[32] T. Seppälä, M. Partinen, A. Penttilä, R. Aspholm, E. Tiainen, and A. Kaukianen, "Sudden death and sleeping history among Finnish men," Journal of Internal Medicine, vol. 229, no. 1, pp. 23-28, 1991.

[33] R. Clay, V. Kapur, J. M. Gronquist et al., "Risk of obstructive sleep apnea in patients with pacemakers and implantable cardioverter defibrillators and incidence of ventricular 
arrhythmia," American Journal of Respiratory and Critical Care Medicine, vol. 193, p. A4206, 2016.

[34] S. Javaheri, "Sleep disorders in systolic heart failure: a prospective study of 100 male patients. The final report," International Journal of Cardiology, vol. 106, no. 1, pp. 21-28, 2006.

[35] T. Bitter, N. Westerheide, C. Prinz et al., "Cheyne-Stokes respiration and obstructive sleep apnoea are independent risk factors for malignant ventricular arrhythmias requiring appropriate cardioverter-defibrillator therapies in patients with congestive heart failure," European Heart Journal, vol. 32, no. 1, pp. 61-74, 2011.

[36] F. Kilicaslan, A. Tokatli, F. Ozdag et al., “Tp-e interval, Tpe/QT ratio, and Tp-e/QTc ratio are prolonged in patients with moderate and severe obstructive sleep apnea," Pacing and Clinical Electrophysiology, vol. 35, no. 8, pp. 966-972, 2012.

[37] I. Topilski, O. Rogowski, R. Rosso et al., "The morphology of the QT interval predicts torsade de pointes during acquired bradyarrhythmias," Journal of the American College of Cardiology, vol. 49, no. 3, pp. 320-328, 2007.

[38] J. Castro Hevia, C. Antzelevitch, F. Tornés Bárzaga et al., "Tpeak-Tend and Tpeak-Tend dispersion as risk factors for ventricular tachycardia/ventricular fibrillation in patients with the Brugada syndrome," Journal of the American College of Cardiology, vol. 47, no. 9, pp. 1828-1834, 2006.

[39] G. Erikssen, K. Liestøl, L. Gullestad et al., "The terminal part of the QT interval (T peak to Tend): a predictor of mortality after acute myocardial infarction," Annals of Noninvasive Electrocardiology, vol. 17, no. 2, pp. 85-94, 2012.

[40] X. Zhao, Z. Xie, Y. Chu et al., “Association between Tp-e/QT ratio and prognosis in patients undergoing primary percutaneous coronary intervention for ST-segment elevation myocardial infarction," Clinical Cardiology, vol. 35, no. 9, pp. 559-564, 2012.

[41] M. Shimizu, H. Ino, K. Okeie et al., "T-peak to T-end interval may be a better predictor of high-risk patients with hypertrophic cardiomyopathy associated with a cardiac troponin I mutation than QT dispersion," Clinical Cardiology, vol. 25, no. 7, pp. 335-339, 2002.

[42] F. Knebel, I. Schimke, K. Pliet et al., "NT-ProBNP in acute heart failure: correlation with invasively measured hemodynamic parameters during recompensation," Journal of Cardiac Failure, vol. 11, no. 5, pp. S38-S41, 2005.

[43] R. Krittayaphong, T. Boonyasirinant, P. Saiviroonporn et al., "Correlation Between NT-pro BNP levels and left ventricular wall stress, sphericity index and extent of myocardial damage: a magnetic resonance imaging study," Journal of Cardiac Failure, vol. 14, no. 8, pp. 687-694, 2008.

[44] Y. Sekiguchi, K. Aonuma, Y. Yamauchi et al., "Chronic hemodynamic effects after radiofrequency catheter ablation of frequent monomorphic ventricular premature beats," Journal of Cardiovascular Electrophysiology, vol. 16, no. 10, pp. 1057-1063, 2005.

[45] H. Tada, S. Ito, G. Shinbo et al., "Significance and utility of plasma brain natriuretic peptide concentrations in patients with idiopathic ventricular arrhythmias," Pacing and Clinical Electrophysiology, vol. 29, no. 12, pp. 1395-1403, 2006.

[46] J. B. Skranes, G. Einvik, S. K. Namtvedt et al., "Biomarkers of cardiovascular injury and stress are associated with increased frequency of ventricular ectopy: a population-based study," BMC Cardiovascular Disorders, vol. 16, p. 233, 2016.

[47] C. F. van Huls van Taxis, S. R. Piers, M. de Riva Silva et al., "Fatigue as presenting symptom and a high burden of premature ventricular contractions are independently associated with increased ventricular wall stress in patients with normal left ventricular function," Circulation: Arrhythmia and Electrophysiology, vol. 8, pp. 1452-1459, 2015.

[48] S. Tasci, R. Manka, S. Scholtyssek et al., "NT-pro-BNP in obstructive sleep apnea syndrome is decreased by nasal continuous positive airway pressure," Clinical Research in Cardiology, vol. 95, no. 1, pp. 23-30, 2006.

[49] R. Strehmel, M. Valo, and C. Teupe, "Natriuretic peptide and high-sensitive troponin $\mathrm{t}$ concentrations correlate with effectiveness of short-term cpap in patients with obstructive sleep apnea and coronary artery disease," Clinical Medicine Insights: Circulatory, Respiratory and Pulmonary Medicine, vol. 10, pp. 33-39, 2016. 


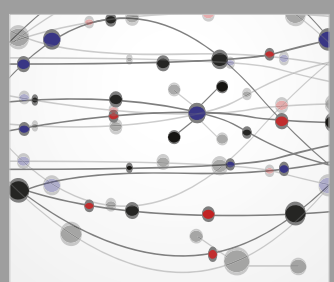

The Scientific World Journal
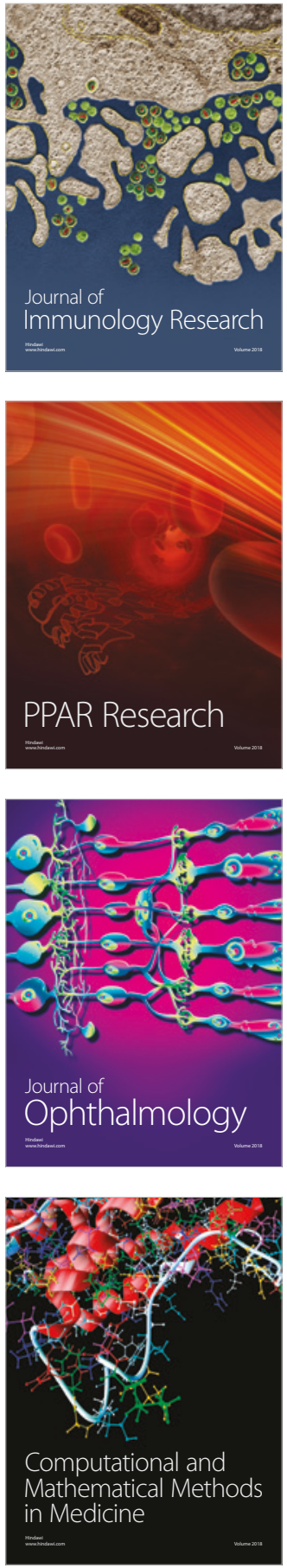

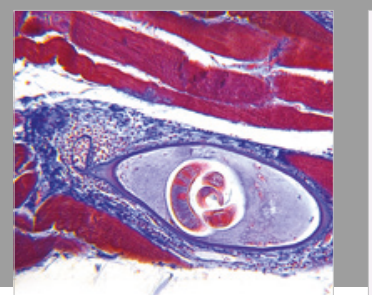

Gastroenterology Research and Practice

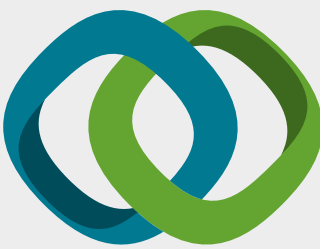

\section{Hindawi}

Submit your manuscripts at

www.hindawi.com
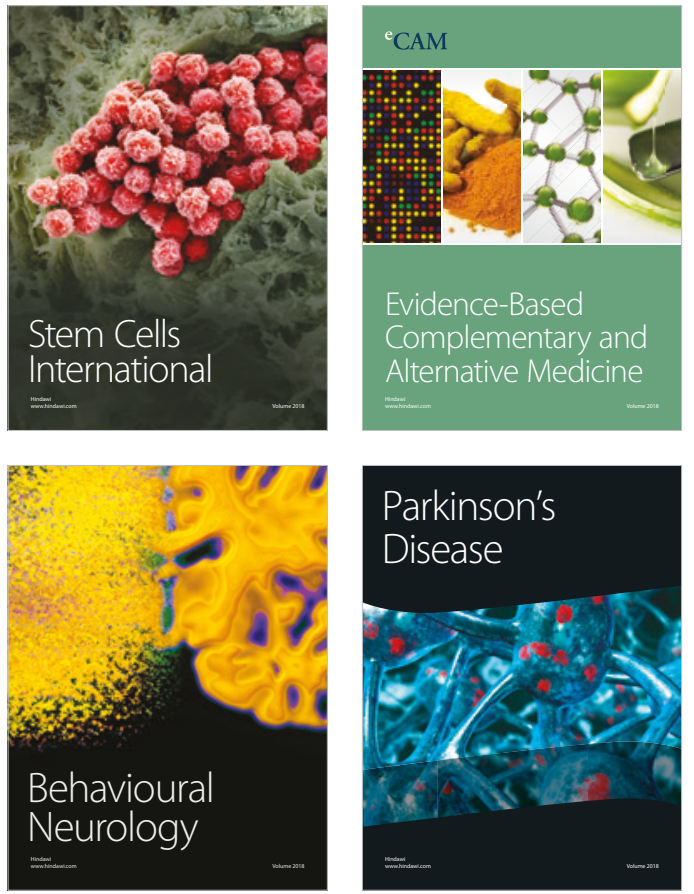

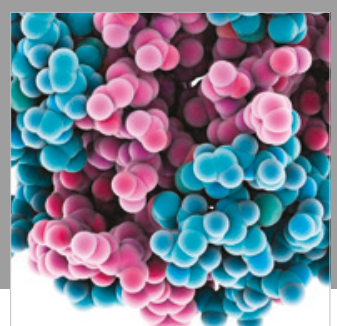

ournal of

Diabetes Research

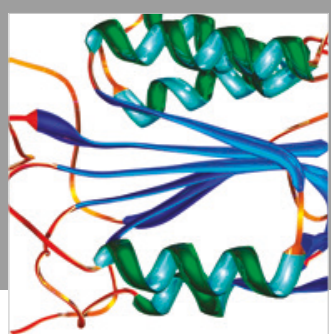

Disease Markers
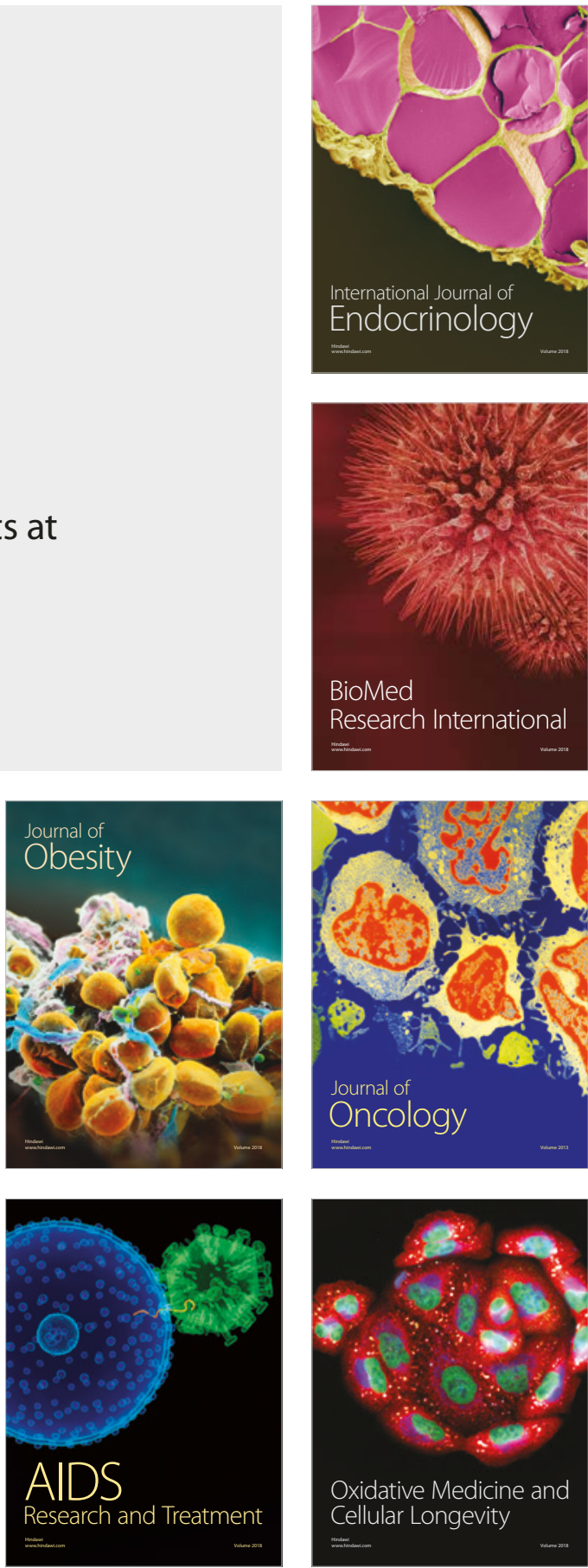